\title{
Crack-front propagation during three-point-bending tests of polymethyl-methacrylate beams
}

\author{
J.A. Loya ${ }^{\text {b,*, E.I. Villa }}{ }^{\mathrm{a}}$, J. Fernández-Sáez ${ }^{\mathrm{b}}$ \\ a Área Departamental Construcciones, Facultad de Ingeniería, Universidad Nacional de La Plata, Calle 48 y 115 s/n, (B1900 TAG) La Plata, \\ Buenos Aires, República Argentina \\ ${ }^{\mathrm{b}}$ Departamento de Mecánica de Medios Continuos y Teoría de Estructuras, Universidad Carlos III de Madrid. Avda. de la Universidad, \\ 30, 28911 Leganés, Madrid, Spain
}

Keywords:

Crack-front propagation

PMMA

Three-point-bending

High-speed camera

\begin{abstract}
A B S T R A C T
Crack front evolution in polymethyl methacrylate (PMMA) beams was measured during quasi static three point bending tests performed on a universal testing machine. A high speed camera was used to record the crack front propagation process through the specimen thickness and to determine the instantaneous crack length during the test, considering the effect of different initial notch lengths and loading point displacement rates. The average steady crack propagation speed was also calculated and correlated with the stored elastic energy, and these results have been compared with those reported by other authors for different test conditions. This experimental technique appears to be suitable to determine the influence of the test conditions on the crack propagation speed of PMMA specimens.
\end{abstract}

\section{Introduction}

A key objective being pursued in dynamic crack prop agation analysis is to understand and predict the path followed by a crack and its velocity during the propagation process. In the one dimensional case, the equation that governs crack propagation in a brittle material can be established by equating the energy release rate at the crack tip, $G_{d}$, that depends on the geometry of the body, the initial crack length, $a$, and its propagation velocity, $\dot{a}$. It must be evaluated through the solution of a corresponding elasto dynamic problem with the dynamic fracture energy of the material, $G_{C}$, which depends on the crack velocity, i.e. [1]:

$$
G_{\mathrm{d}}(a, \dot{a}, \ldots) \quad G_{\mathrm{c}}(\dot{a}, \ldots)
$$

A phenomenological rate dependant fracture energy, $G_{\mathrm{c}} \quad G_{\mathrm{c}}(\dot{a})$ for each material is broadly accepted [2].

\footnotetext{
* Corresponding author. Tel.: +34 916248880; fax: +34 916249973 . E-mail address: jloya@ing.uc3m.es (J.A. Loya).
}

One of the most important difficulties for the determi nation of $G_{c}(\dot{a})$ lies in the measurement of the crack velocity. In particular, dynamic crack propagation in poly mers has been investigated using different optical methods such as photoelasticity [2 6] and the method of caustics [7 11], that allow tracking of the crack tip evolution on the surface of the specimen.

In a recent work, Murphy and Ivankovic [12] presented results of the crack propagation speed in PMMA plates $8 \mathrm{~mm}$ thick with a small notched edge in tension.

On the other hand, Zhou et al. [13] have also conducted tests on rectangular PMMA plates of $3 \mathrm{~mm}$ thickness. The plates were pre stressed to a certain constant load when a small crack was induced in the middle of the plate, the speed of which was measured by bands being crossed during the propagation process.

However, the crack propagation speed has not been measured in three point bending. This has great interest because this test configuration is extensively used to determine the fracture properties of a wide range of materials. 


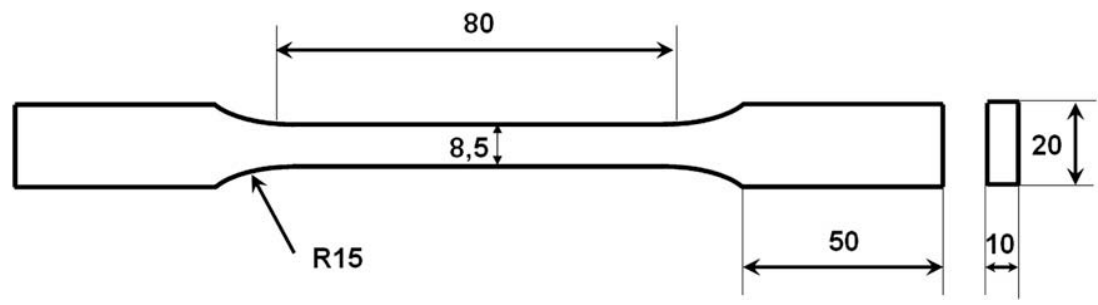

Fig. 1. Geometry of the tension test specimens. Dimensions in $\mathrm{mm}$.

In this work, the crack propagation velocity in PMMA specimens with different initial notch lengths was measured in quasi static three point bending tests per formed on a universal testing machine at different loading point displacement rates. The crack propagation process was recorded using high speed photographic equipment.

From the instantaneous crack length, the propagation velocity was also calculated and correlated with the stored elastic energy, and the results were compared with those reported by other authors for different test conditions. This experimental technique appears to be suitable to observe the crack propagation process and to analyse the influence of the test conditions on the crack propagation velocity.

The information gained from these tests enabled the relationship between the softening function of the material with the crack propagation speed to be determined, knowledge of which is key when using cohesive zone models to analyse this type of material.

\section{Experimental technique}

\subsection{Uniaxial tension tests}

The material tested was a glassy, commercially available polymethyl methacrylate (PMMA), which is generally regarded as quasi brittle and linearly elastic.

Uniaxial tensile yield tests were performed to establish the stress strain curve and the values of Young's modulus, $E$, yield strength, $\sigma_{0.2}$ and resistance, $\sigma_{\mathrm{u}}$, of the material. The geometry of the specimens is schematically shown in Fig. 1.

Specimens were loaded by a computer controlled INS TRON 8516 universal testing machine under displacement control mode at room temperature, and the loading speed was set at a constant rate of $0.25 \mathrm{~mm} / \mathrm{min}$.

\subsection{Fracture toughness tests}

Following the standard ASTM D 5045 [14], the fracture toughness of the material, $K_{\mathrm{IC}}$, was determined by three point bending test (TPB) using cracked specimens with the geometry presented in Fig. 2, where $L, B$ and $W$ are, respectively, the length, the thickness and the width of the specimen, $S$ is the span, and $a_{0}$ is the initial notch length.

Specimens used to obtain the fracture toughness were fatigued using a resonance fatigue machine [15] that allowed control of the crack growth from an initial notch of $a_{0} \quad 8.5 \mathrm{~mm}$ length.

The mechanical properties determined for PMMA, as well as the fracture toughness, are shown in Table 1.

Crack front propagation was analysed on three point bending specimens, considering different initial notch lengths ( $a_{0} \quad 2,5,8$ and $10 \mathrm{~mm}$ ). Notches were machined using a diamond sawing wire, creating a $0.28 \mathrm{~mm}$ semi circular notch tip radius. The bending tests were per formed on the same universal testing machine under displacement control mode for different loading rates $(0.25,0.5,2.0$ and $5.0 \mathrm{~mm} / \mathrm{min}$ ) (see Fig. 3).

During the tests, due to the optical properties of the PMMA, the crack front propagation was recorded using a high speed camera Photron Ultima APX focusing the crack propagation plane at approximately a $45^{\circ}$ angle. The lighting of the test was controlled by an Arrisun 12 Plus lamphead with a $1200 \mathrm{~W}$ Hydrargyrum Medium arc Iodide (HMI) lamp. A scheme of the position of the different equipment used in the tests is presented in Fig. 4.

Table 1

Mechanical properties of PMMA.

\begin{tabular}{llll}
\hline$E(\mathrm{MPa})$ & $\sigma_{0.2}(\mathrm{MPa})$ & $\sigma_{\mathrm{u}}(\mathrm{MPa})$ & $K_{\mathrm{IC}}\left(\mathrm{MPa} \mathrm{m}^{\wedge}{ }^{0.5}\right)$ \\
\hline 2870 & 27.1 & 47.8 & 1.05 \\
\hline
\end{tabular}

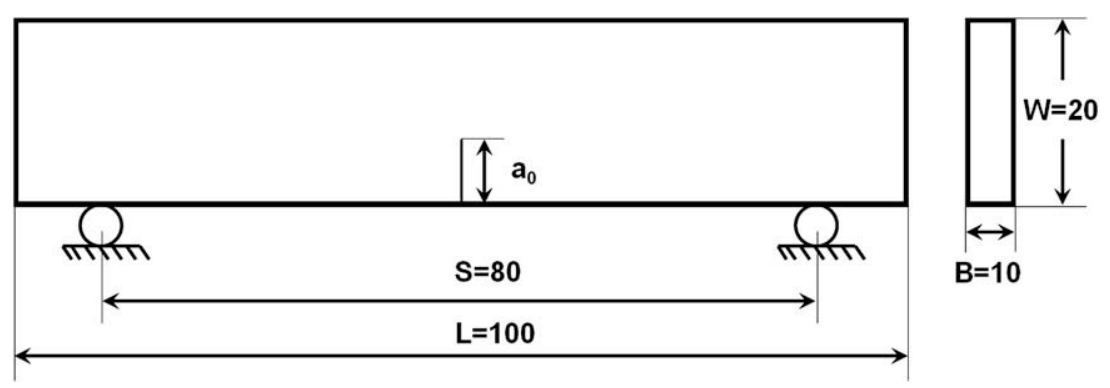

Fig. 2. General outline of the three-point-bending-test specimens. Dimensions in mm. 


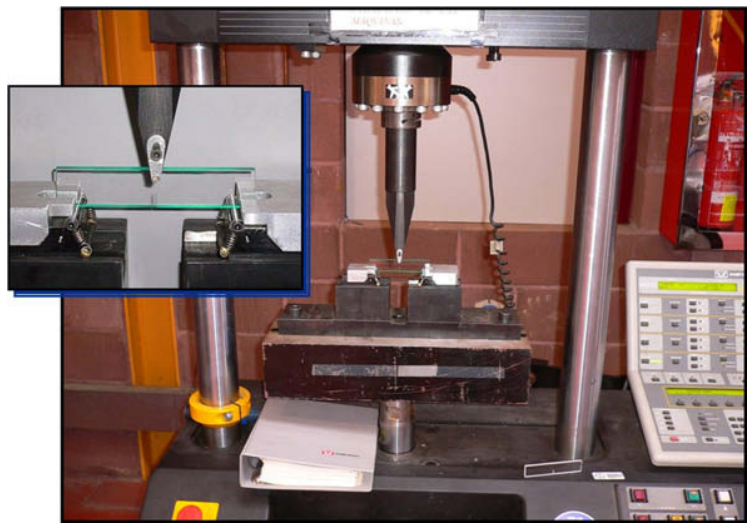

Fig. 3. Three-point-bending test arrangement

Through this technique, a sequence of pictures of the crack front propagation process is obtained in every test, as presented in Fig. 5.

In order to analyze the sequence of pictures obtained, an ad hoc image processing code based on MatlLab has been developed. This code recognizes the crack propagation zone, where the discontinuity in the material appears as a darker zone in comparison with the intact zone. This distinction enables the image processing code to clearly distinguish the cracked zone, determining the crack front at any time, $t$, and the crack length at different planes $z$ through the thickness $(z / B \quad 0,0.25,0.50,0.75$ and 1$)$ (Fig. 6). From those values, a representative thickness

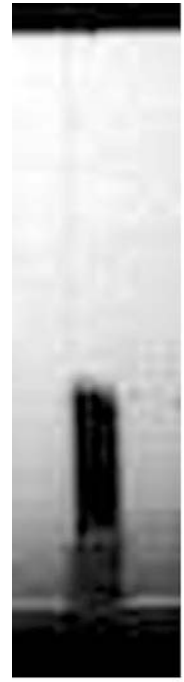

a

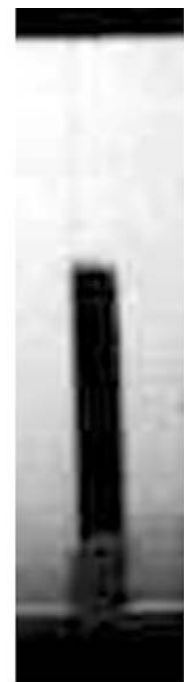

b

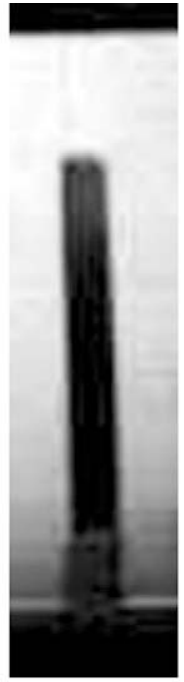

C

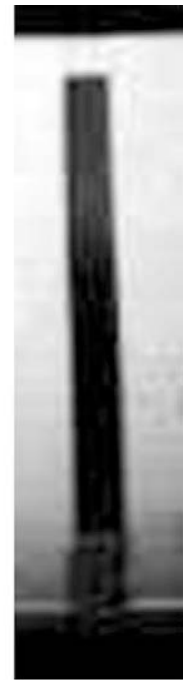

d
Fig. 5. Sequence of the process of propagation of the crack: a) $t \quad 33 \mu \mathrm{s}$; b) $t \quad 55 \mu \mathrm{s}$; c) $t \quad 77 \mu \mathrm{s}$; d) $t \quad 100 \mu \mathrm{s}$.

average crack length, $\bar{a}(t)$ is obtained, the time history of which is presented in Fig. 7.

From the crack length $\bar{a}(t)$ provided by the image pro cessing code, the instantaneous thickness average crack propagation velocity, $\dot{a}(t)$, was evaluated as follows:

$\dot{a}(t) \quad \frac{\bar{a}(t+\Delta t) \quad \bar{a}(t \quad \Delta t)}{2 \Delta t}$

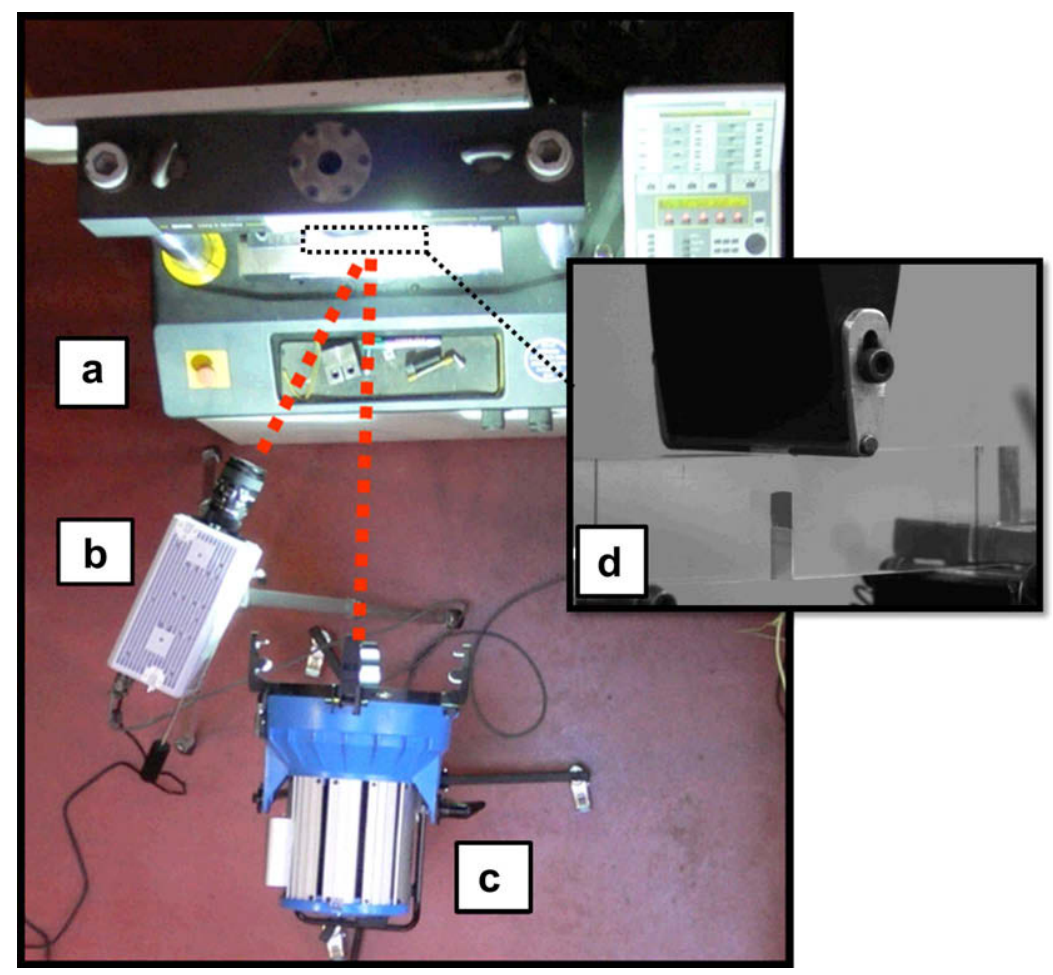

Fig. 4. Location of equipment during test: a) universal testing machine; b) high-speed camera; c) spot light; d) specimen. 


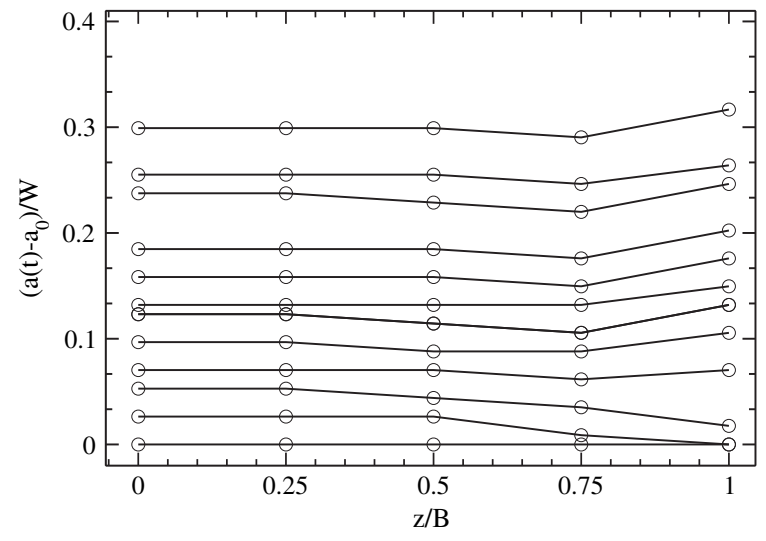

Fig. 6. Nondimensional crack-front extension through the thickness.

$\Delta t$ being the interval of time between two consecutive pictures.

Load displacement records were determined from the tests as well as the maximum load (an example of these records is presented in Fig. 8). Once the maximum load is reached, the crack begins to propagate and, therefore, the available energy for crack propagation, $G_{\mathrm{c}}$, can be deter mined from the area under the load displacement curve, $A$, as follows:

$$
G_{c} \quad \frac{2 A}{B\left(W \quad a_{0}\right)}
$$

In this work, 16 different cases were analysed, corre sponding to combinations of four different notch lengths $(2,5,8$ and $10 \mathrm{~mm})$ and four loading rates $(0.25,0.5,2.0$ and $5.0 \mathrm{~mm} / \mathrm{min})$.

\section{Results}

The instantaneous crack propagation velocity, $\dot{a}$, versus the crack length evolution measured at different loading rates is presented in Figs. 9 12, for different initial notch lengths. These figures reflect that the crack propagation velocity decreases significantly as long as the initial notch

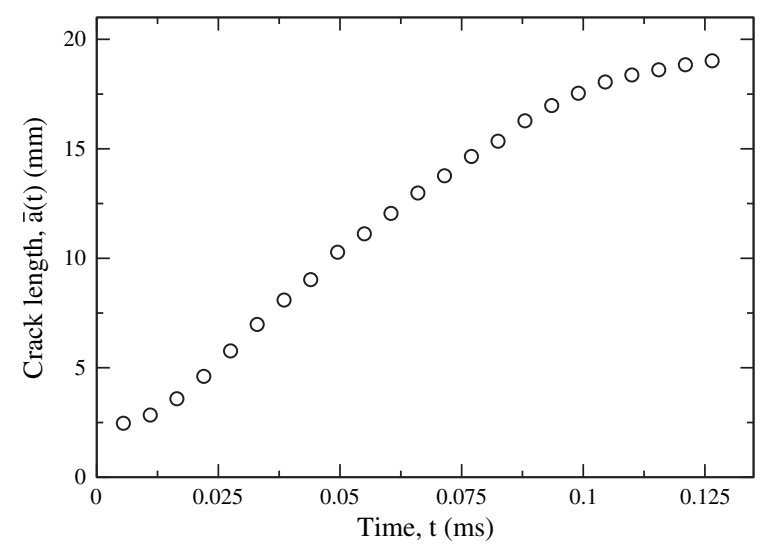

Fig. 7. Average crack-length versus time.

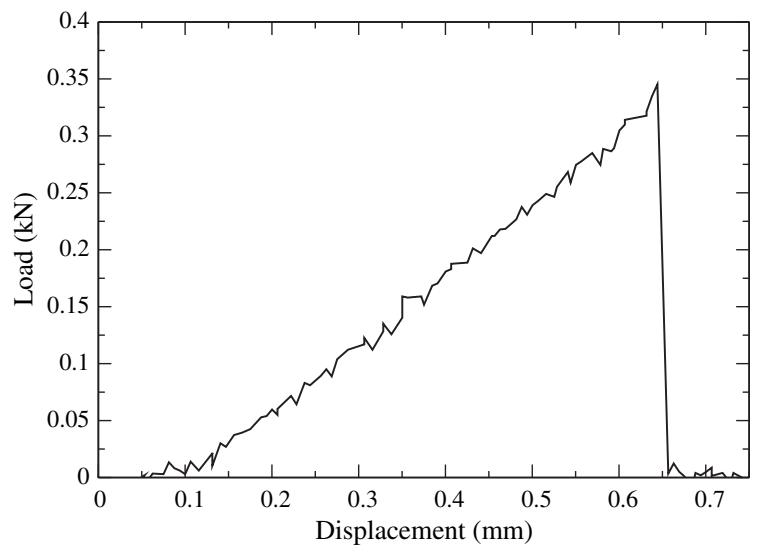

Fig. 8. Load-displacement record.

increases, with sudden changes in speed for the longest initial notch, $a_{0} 10 \mathrm{~mm}$, which virtually nullify the prop agation velocity at some intermediate points of the test (Fig. 12).

For each test, an average steady crack propagation speed, $\bar{v}$, was calculated by the following expression:

$\bar{v} \quad \frac{a_{\mathrm{f}}}{a_{0}}$

where $a_{0}$ is the initial notch length, $a_{\mathrm{f}}$ is the final crack length reached during the test, and $t_{0}$ and $t_{\mathrm{f}}$ the corre sponding times.

The average speed thus calculated was very similar to what would have been determined as the slope of the regression line of the crack length with respect to the time.

In Fig. 13, the evolution of the average speed with respect to the crack length is represented for each of the four test velocities. These results do not show a clear effect of test velocity on the average speed of the crack propagation.

Fig. 14 shows the experimental data obtained for $G_{\mathrm{C}}$ versus the average steady crack speed $\bar{v}$.

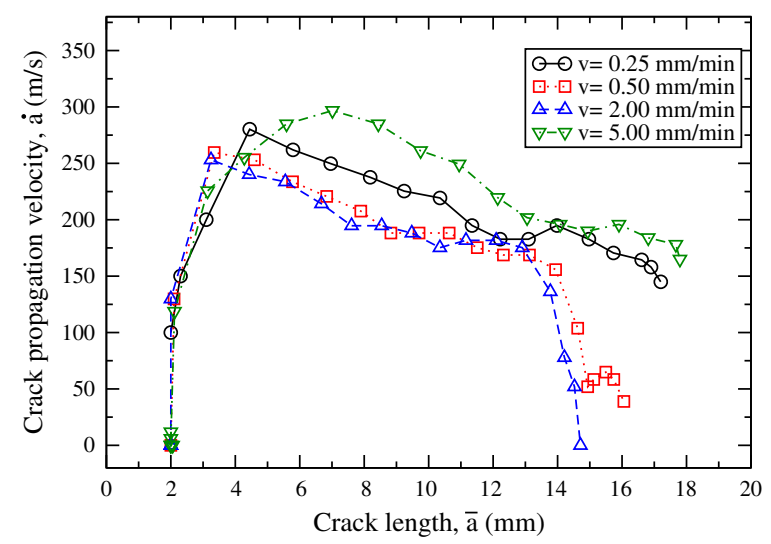

Fig. 9. Instantaneous crack-propagation velocity depending on the cracklength: $a_{0} \quad 2 \mathrm{~mm}$. 


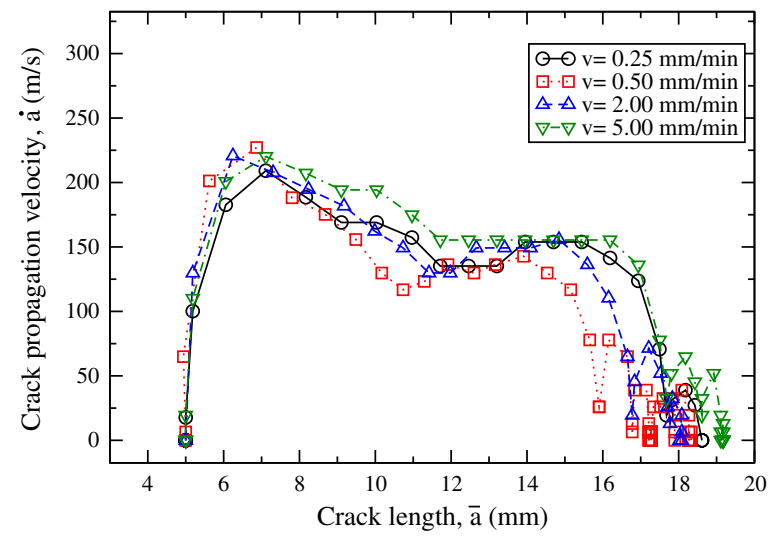

Fig. 10. Instantaneous crack-propagation velocity depending on the cracklength: $a_{0} \quad 5 \mathrm{~mm}$.

Zhou et al. [13] have proposed an empirical relation to correlate these two variables in the range of speeds between $100 \mathrm{~m} / \mathrm{s}$ and $650 \mathrm{~m} / \mathrm{s}$, given by:

$$
G_{\mathrm{c}}(\bar{v}) \quad G_{0} \operatorname{Ln}\left(\frac{v_{\mathrm{L}}}{v_{\mathrm{L}} \bar{v}}\right)
$$

where the constants $G_{0}$ and $v_{\mathrm{L}}$ take the following values: $G_{0} \quad 2000 \mathrm{~N} / \mathrm{m}$ and $v_{\mathrm{L}} \quad 675 \mathrm{~m} / \mathrm{s}$. Using this correlation in our tests, where the speed varied between $60 \mathrm{~m} / \mathrm{s}$ and $200 \mathrm{~m} / \mathrm{s}$, it does not fit the experimental data as can be seen in Fig. 14. Therefore, if we recalculated the $G_{0}$ value, we can observe in the figure that the correlation described above for $G_{0} \quad 6825 \mathrm{~N} / \mathrm{m}$ reproduces the experimental results with good accuracy.

To explain this discrepancy it is necessary to note that a radiused notch is considered in the results shown here, rather than a sharp crack [13]. To account for the latter, instead of the fracture toughness we should consider the apparent toughness, $K_{\mathrm{Q}}$, obtained by testing a specimen with the same dimensions used to measure $K_{\mathrm{IC}}$ but with a notch instead of a crack. A relationship similar to Eq. (5) is proposed maintaining the value of $v_{\mathrm{L}} 675 \mathrm{~m} / \mathrm{s}$, but replacing the value of $G_{0}$ by $G_{0}^{*}$, as follows:

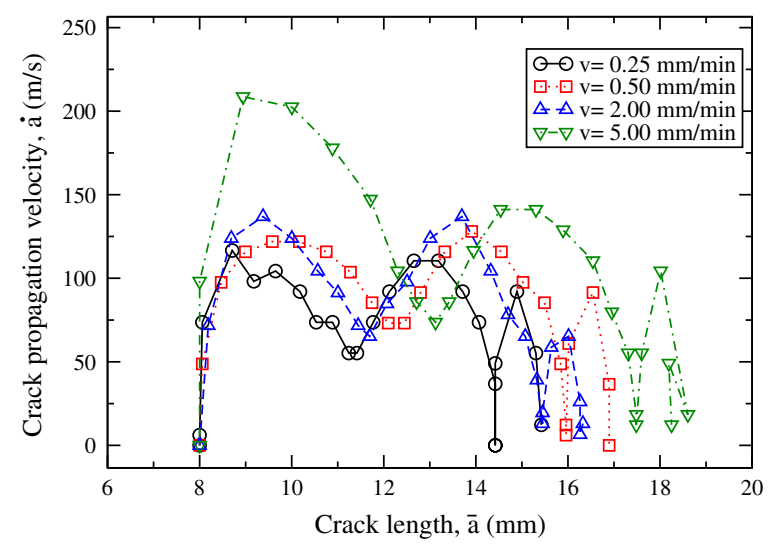

Fig. 11. Instantaneous crack propagation velocity depending on the cracklength: $a_{0} \quad 8 \mathrm{~mm}$.

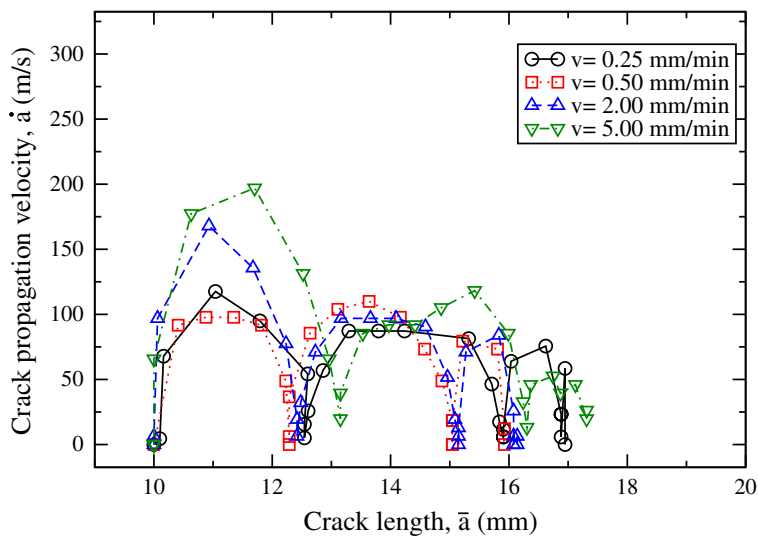

Fig. 12. Instantaneous crack-propagation velocity depending on the cracklength: $a_{0} 10 \mathrm{~mm}$.

$G_{0}^{*} \quad G_{0}\left(\frac{K_{Q}}{K_{I C}}\right)^{2}$

where the value of the apparent resulting toughness $\left(K_{\mathrm{Q}} \quad 1,85 \mathrm{MPa} \mathrm{m}^{0.5}\right)$ and the fracture toughness reported in Table 1, yields a value of $G_{0}^{*}$ equal to $6208 \mathrm{~N} / \mathrm{m}$.

The correlation (Eq. (5)) in our range of speeds for cracked specimens fits the data with good accuracy.

\section{Summary and concluding remarks}

In this work, the crack propagation velocity in PMMA beams with different initial notch lengths $\left(a_{0} \quad 2,5,8\right.$ and $10 \mathrm{~mm}$ ) was measured in quasi static three point bending tests.

The material chosen for testing is a glassy, commercially available polymer which is generally considered quasi brittle and linearly elastic under tension at room temperature.

Specimens were loaded by a computer controlled INS TRON 8516 universal testing machine in control displace ment mode at different loading point displacement rates $(0.25,0.5,2.0$, and $5.0 \mathrm{~mm} / \mathrm{min})$.

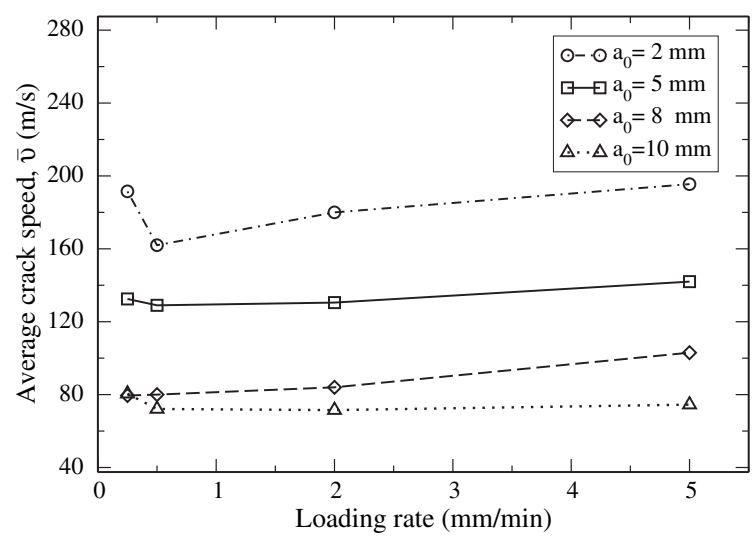

Fig. 13. Average crack speed of propagation with respect to the load-rate. 


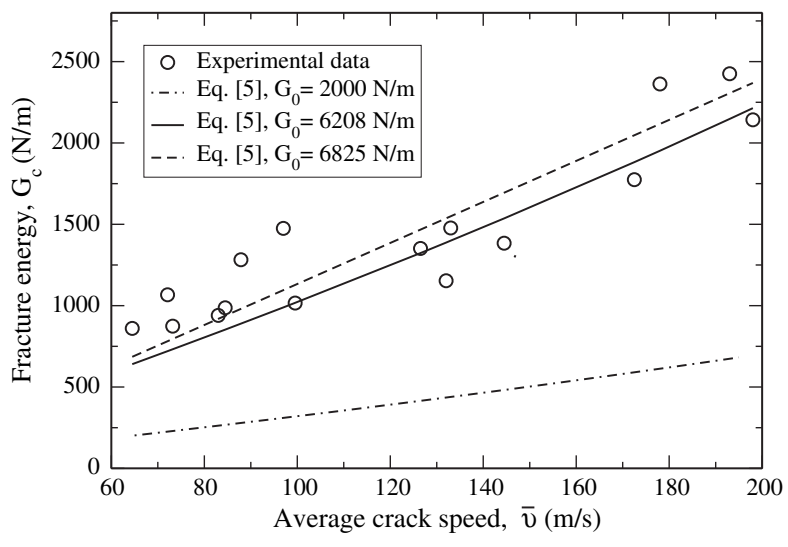

Fig. 14. Fracture energy with respect to the average steady crack-propagation speed.

A new experimental optical methodology to record the crack propagation process by means of a high speed camera was presented. A particular feature of this optical methodology in comparison with others, for example photoelasticity or caustics, is that the crack length can be measured at different points through the thickness at any time. From these values, a thickness average crack length and velocity were obtained.

This experimental technique appears to be suitable to determine the influence of the test conditions in the crack propagation speed of PMMA specimens.

From the results obtained through this technique, it is apparent that in the range of the loading rates considered in this work, the notch length is the parameter that most affects the speed of propagation of the crack, reducing the speed as the notch lengthens.

The instantaneous crack length during the test was also determined and the average steady crack propagation speed was calculated and correlated with the stored elastic energy.

The experimental results obtained for the fracture energy with respect to the average crack speed can be accurately modelled by the empirical correlation reported by the Zhou et al. [13] extended to our range of speeds and using a proposed modification to consider notched instead of cracked specimens.

\section{Acknowledgment}

This research was carried out with the financial support of the Universidad Carlos III de Madrid and of the Comu nidad Autonoma de Madrid under Project CCG08 UC3M/ MAT 4464.

\section{References}

[1] L.B. Freund, Dynamic Fracture Mechanics. Cambridge University Press, 1990.

[2] J.W. Dally, W.L. Fourney, G.R. Irwin, On the uniqueness of the stress intensity factor-crack velocity relationship. International Journal of Fracture 27 (1985) 159-168.

[3] A.S. Kobayashi, M. Ramulu, M.S. Dadkhah, K.-H. Yang, B.S.J. Kang, Dynamic fracture toughness. International Journal of Fracture 30 (1986) 275-285.

[4] A. Shukla, H. Nigam, A note on the stress intensity factor and crack velocity relationship for Homalite 100. Engineering Fracture Mechanics 25 (1986) 91-102.

[5] J.W. Dally, R.K. Agarwal, R.J. Sanford, A study of hysteresis in the $K_{\text {ID }}$ a relation. Experimental Mechanics 30 (1990) 177-183.

[6] A.J. Rosakis, Two optical techniques sensitive to gradients of optical path difference: the method of caustics and the coherent gradient sensor (CGS). in: J.S. Epstein (Ed.), Experimental Techniques in Fracture. VCH Publishers, 1993, pp. 327-425.

[7] T. Kobayashi, J.W. Dally, Relation between crack velocity and the stress intensity factor in birefringent polymers ASTM STP 627. in: G.T. Hahn, M.F. Kanninen (Eds.), Fast Fracture and Crack Arrest (1977), pp. 257-273.

[8] P.S. Theocaris, G.A. Papadopoulos, Elastodynamic forms of caustics for running cracks under constant velocity. Engineering Fracture Mechanics 13 (1980) 683-698.

[9] W.G. Knauss, K. Ravi-Chandar, Some basic problems in stress wave dominated fracture. International Journal of Fracture 27 (1985) 127-143.

[10] X.F. Yao, W. Xu, M.Q. Xu, K. Arakawa, T. Mada, K. Takahashi, Experimental study of dynamic fracture behavior of PMMA with overlapping offset-parallel cracks. Polymer Testing 22 (2003) 663-670.

[11] T. Nishioka, T. Murakami, H. Uchiyama, K. Sakakura, H. Kittaka, Specimen size effects on dynamic crack propagation and arrest in DCB specimens. Engineering Fracture Mechanics 39 (1991) 757-767.

[12] N. Murphy, A. Ivankovic, The prediction of dynamic fracture evolution in PMMA using a cohesive zone model. Engineering Fracture Mechanics 72 (2005) 861-875.

[13] F. Zhou, J.F. Molinari, T. Shioya, A rate-dependent cohesive model for simulating dynamic crack propagation in brittle materials. Engineering Fracture Mechanics 72 (2005) 1383-1410.

[14] Astm D 5045-96, Standard Test Method for Plane-Strain Fracture Toughness and Strain Energy Release Rate of Plastic Materials. American Society for Tensting and Materials, Philadelphia, 1996.

[15] J.A. Loya, Efectos tridimensionales en la determinacion de la tenacidad de fractura dińamica, Ph.D. Thesis, University Carlos III of Madrid, 2004. 